\title{
Differentiating benign from malignant cardiac tumors with cardiac magnetic resonance imaging
}

\author{
Mahwash Kassi, MD, ${ }^{\mathrm{a}}$ Venkateshwar Polsani, MD, ${ }^{\mathrm{b}}$ Robert C. Schutt, MD, ${ }^{\mathrm{c}}$ Solomon Wong, MS,
} Faisal Nabi, MD, ${ }^{\mathrm{a}}$ Michael J. Reardon, MD, ${ }^{\mathrm{a}}$ and Dipan J. Shah, MD ${ }^{\mathrm{a}}$

\section{ABSTRACT}

Background: The purpose of this analysis is to describe the differences in cardiac magnetic resonance characteristics between benign and malignant tumors, which would be helpful for surgical planning.

Methods: This was a prospective cohort study of 130 patients who underwent cardiac magnetic resonance imaging for evaluation of a suspected cardiac mass. After excluding thrombi and tumors without definitive diagnosis, 66 tumors were evaluated for morphologic features and tissue composition.

Results: Of the 66 patients, $39(59.0 \%)$ had malignant tumors and $27(41.0 \%)$ had benign tumors. Patients with malignant tumors were younger when compared with those with benign tumors (age 51 years [42.8-60.0] vs 65 years [60.0-71.0] median). Malignant tumors more often demonstrated tumor invasion (69\% vs $0 \%$ $P<.001)$ and were more often associated with pericardial effusion $(41 \%$ vs $7.4 \%$ $P=.004)$. Presence of first-pass perfusion $(100 \%$ vs $33 \% P<.001)$ and late gadolinium enhancement $(100 \%$ vs $59.2 \%, P<.001)$ were significantly higher in malignant tumors. In logistic regression modeling, tumor invasion $(P<.001)$ and first-pass perfusion $(P<.001)$ were independently associated with malignancy. Furthermore, using classification and regression tree analysis, we developed a decision tree algorithm to help differentiate benign from malignant tumors (diagnostic accuracy $\sim 90 \%$ ). The algorithm-weighted cost of misclassifying a malignant tumor as benign was twice that of classifying a benign tumor as malignant.

Conclusions: Our study demonstrates that cardiac magnetic resonance imaging is a useful noninvasive method for differentiating malignant from benign cardiac tumors. Tumor size, invasion, and first-pass perfusion were useful imaging characteristics in differentiating benign from malignant tumors. ( $\mathrm{J}$ Thorac Cardi-

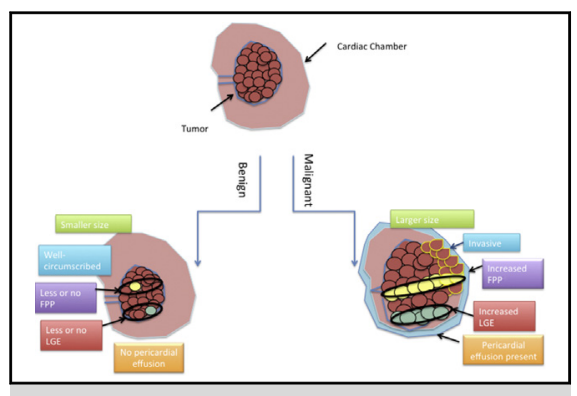

Illustration demonstrating differences between benign and malignant tumors based on our proposed algorithm. FPP, First-pass perfusion; LGE, late gadolinium enhancement.

\section{Central Message}

CMR imaging, particularly of tumor invasion and FPP, is valuable for differentiating benign from malignant tumors.

\section{Perspective}

Not all cardiac tumors are amenable to biopsy. CMR imaging can play a key role in differentiating benign from malignant tumors to aid in appropriate surgical planning. Our study results suggest that invasive tumors and increased FPP are suggestive of malignant cardiac tumors.

See Commentary page 1923. ovasc Surg 2019;157:1912-22)

Primary cardiac tumors are a rare entity, with an estimated prevalence of $0.002 \%$ to $0.3 \% .^{1,2}$ It is estimated that one quarter of all cardiac tumors are malignant. Surgical resection is the only option for treatment in most cases

From the ${ }^{\mathrm{a}}$ Houston Methodist DeBakey Heart and Vascular Center, Houston, Tex; ${ }^{\mathrm{b}}$ Piedmont Heart Institute, Atlanta, Ga; and ${ }^{\mathrm{c}}$ Memorial Hermann Medical Group, Houston, Tex.

Date and number of Institutional Review Board approval: Institutional Review Board Study \#Pro00001568, approved March 27, 2008.

Received for publication March 15, 2017; revisions received Sept 2, 2018; accepted for publication Sept 14, 2018; available ahead of print Dec 11, 2018.

Address for reprints: Dipan J. Shah, MD, Houston Methodist DeBakey Heart and Vascular Center, 6550 Fannin St, \#677, Houston, TX 77030 (E-mail: djshah@ houstonmethodist.org).

$0022-5223 / \$ 36.00$

Copyright (c) 2018 by The American Association for Thoracic Surgery

https://doi.org/10.1016/j.jtcvs.2018.09.057 and accounts for 1 in every 500 cardiac surgeries performed. ${ }^{3}$ The surgical technique and complexity may vary depending on the nature of the tumor because malignant tumors have a high risk of local recurrence. Standard resection techniques may be sufficient for benign tumors, but a more aggressive approach including neoadjuvant chemotherapy and radical surgical resection, sometimes even involving cardiac autotransplantation, may be needed for malignant tumors. ${ }^{4}$ Therefore,

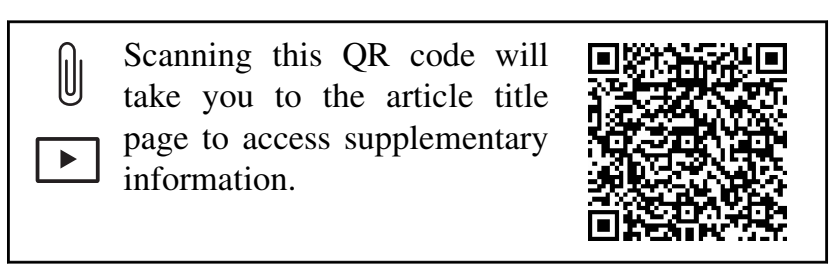




\section{Abbreviations and Acronyms \\ CART $=$ classification and regression tree \\ $\mathrm{CMR}=$ cardiac magnetic resonance \\ FPP $=$ first-pass perfusion \\ LGE = late gadolinium enhancement \\ SSFP $=$ steady-state free precision \\ TSE $=$ turbo spin echo}

differentiating benign from malignant tumors is important for management and surgical planning. Planning for potential radical surgical resection is important both in surgical planning and in appropriate patient consent. Unfortunately, not all tumors are amenable to biopsy, and consequently there is an important role for accurate imaging assessment to help define benign versus malignant tumors (Video 1). ${ }^{5}$

Cardiac magnetic resonance (CMR) is emerging as a robust noninvasive technique for the evaluation of cardiac masses. Cine steady-state free precision (SSFP) sequences help identify the morphology and mobility of the mass. T1- and T2-weighted and proton density-weighted sequences help identify size, extension, and composition of mass. Tissue vascularization can be seen on first-pass perfusion (FPP) imaging, whereas late gadolinium enhancement (LGE) of the lesion helps in characterizing fibrotic components. ${ }^{6,7}$ A broad-based differential for a cardiac mass includes thrombus, benign tumor, and malignant tumor. Because differentiation of tumor from thrombi is well established by CMR, we focus this study on use of CMR for differentiation between benign and malignant tumors. ${ }^{8,9}$

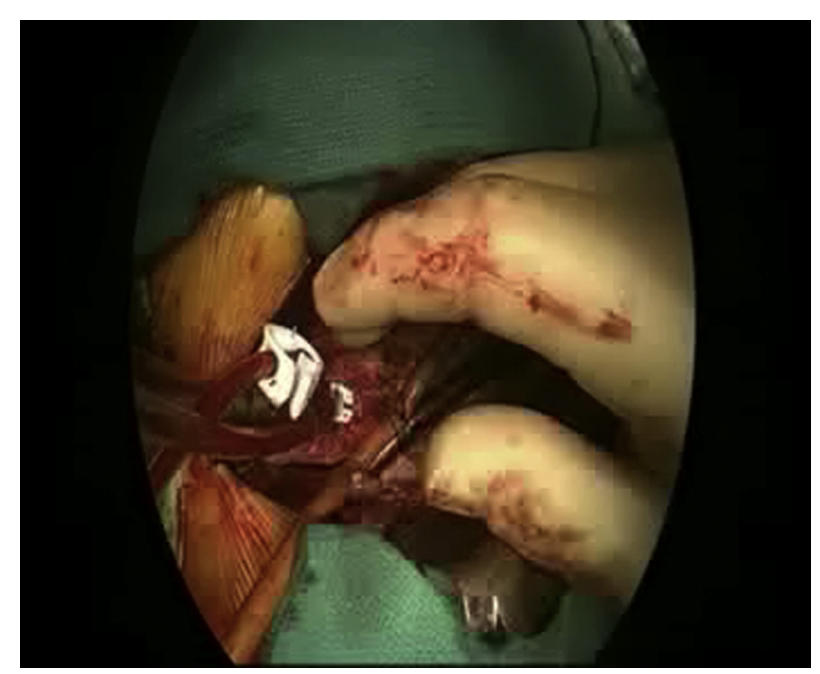

VIDEO 1. Video illustrating surgical resection of the left atrial malignant tumor (sarcoma) and autotransplantation. Video available at: https://www. jtcvs.org/article/S0022-5223(18)32561-3/fulltext.
In this article, we describe the imaging characteristics of patients who presented for CMR tumor evaluation. Our aim was to provide an algorithm to aid in differentiation of benign from malignant tumors.

\section{MATERIALS AND METHODS}

Consecutive patients referred to Houston Methodist DeBakey Heart \& Vascular Center (Houston, Tex) for cardiac tumor evaluation were prospectively enrolled into our CMR registry over a period of 3 years between 2009 and 2012, and a second cohort of patients presenting to Piedmont Heart Institute (Atlanta, Ga) between 2013 and 2015 at Piedmont Heart Center in Atlanta, Georgia, were prospectively enrolled. A total of 130 patients were recruited for the purpose of the study, 9 of whom were from Piedmont Heart Center (Figure 1). Institutional Review Board approval at both institutions was obtained for this study, and all patients provided written informed consent.

All patients who were referred to the CMR laboratory for the evaluation of suspected cardiac tumor seen on other imaging modalities including echocardiogram or CT were included in this study. The studies were read by cardiologists with Level III certification in CMR.

Patients with characteristic CMR features of thrombi (Weinsaft and colleagues, ${ }^{10}$ as detailed next) or patients in whom no final diagnosis for the mass could be elucidated (unknown category) were excluded from the study. Patients were in the unknown category if there were insufficient clinical data to support diagnosis of the tumor (typically no tumor resection). The differentiation of malignant and benign tumors was strictly based on pathology.

Papillary fibroelastoma was excluded because they are usually small and contrast analysis can be challenging. ${ }^{11}$ The best imaging modality for papillary fibroelastomas is echocardiography. ${ }^{12}$ Given distinct characteristics of lipomas and lipomatous hypertrophy, described next, we did not exclude them from our analysis.

\section{Image Acquisition}

CMR images were acquired using a 1.5-T Siemens Avanto or 3.0-T Siemens Verio (Siemens Medical Solutions, Erlangen, Germany) scanner using dedicated phased-array surface coils. Initially, a general survey of the chest was performed using an electrocardiogram-gated dark blood "single shot" fast spin echo and bright blood SSFP sequence in the axial, sagittal, and coronal planes (slice thickness $6 \mathrm{~mm}$ with no gap to cover the entire thorax). This was followed by a full set of electrocardiogram-gated short- and long-axis SSFP cines to cover the entire left ventricle with additional views acquired to delineate the morphologic characteristics of the tumor. (*Typical imaging parameters: TE $1.5 \mathrm{msec}$, spatial resolution $1.5 \times 1.5 \mathrm{~mm}$ in plane, 4-6 mm slice thickness; temporal resolution 30-40 msec.) Tissue characterization of the cardiac mass was performed using (1) electrocardiogram-gated T1-weighted turbo spin echo (TSE) and T2-weighted TSE; (2) first-pass perfusions imaging in at least 2 orthogonal planes to characterize the mass and myocardium using a saturation recovery gradient-recalled echo sequence during a bolus of $0.15 \mathrm{mmol} / \mathrm{kg}$ of Gadopentetate Dimeglumine (Magnevist Bayer HealthCare, LLC, Whippany, NJ) infused at $4 \mathrm{~mL} / \mathrm{s}$; (3) LGE imaging was performed 15 minutes postcontrast injection, using a breath-hold inversion-recovery gradient-echo sequence with inversion time to "null" normal myocardium; (4) long inversion time (TI600) inversion recovery SSFP images were acquired for identification of thrombus. ${ }^{10,12}$ All sequences were performed on all patients.

\section{Image Interpretation}

Morphologic features. General tumor characteristics, including size, location, borders, infiltration, and presence of pericardial effusion, were recorded. A tumor is defined as infiltrative if there is invasion of tumor 


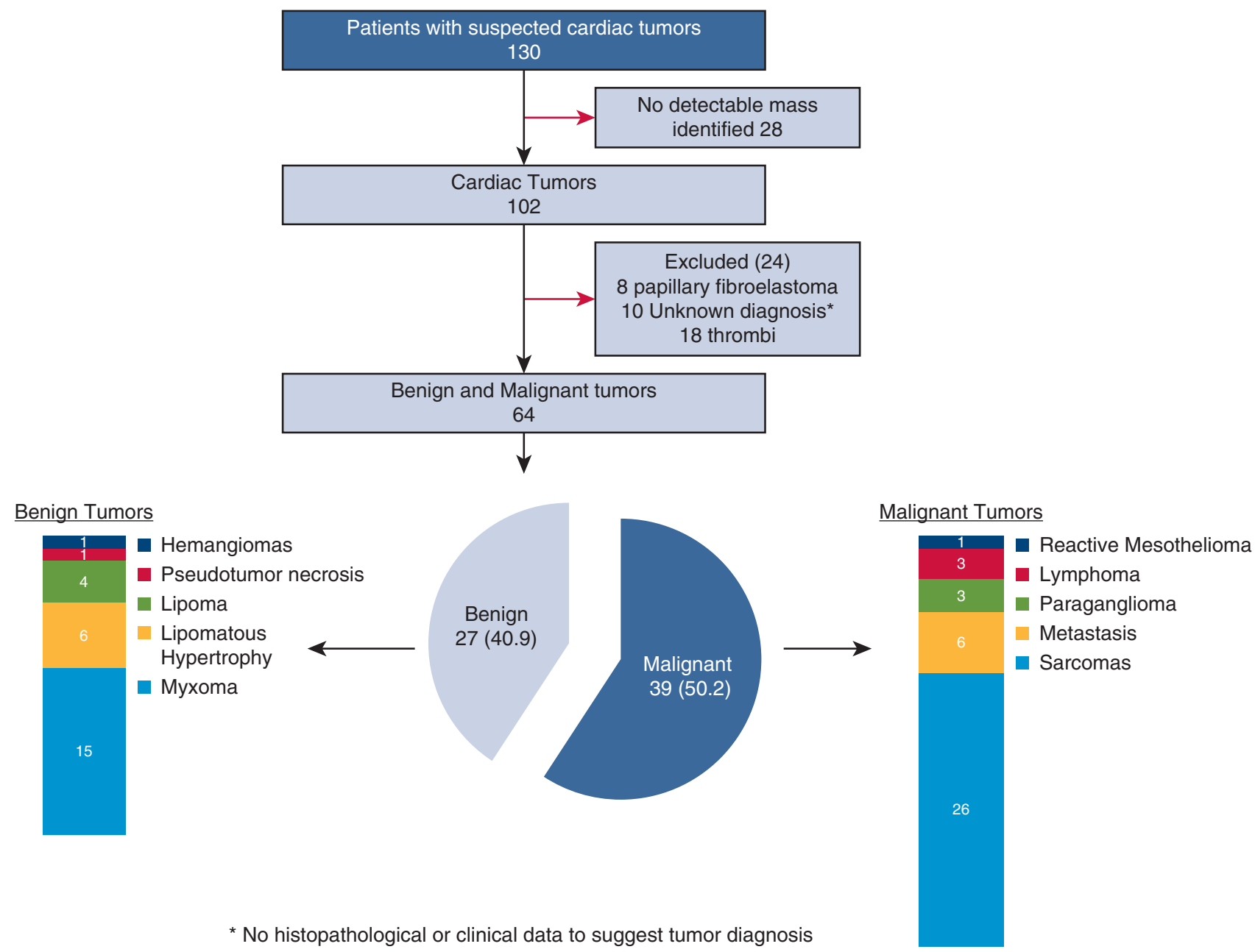

FIGURE 1. Flow sheet for patient population and pie-chart with tumor distribution for benign and malignant tumors.

in the neighboring wall or adjoining structures. As part of our protocol, we identified and collected prior hypothesized features for differentiating malignant from benign cardiac tumors, including origin from right side, size $(>5 \mathrm{~cm})$, and invasion of walls and adjacent chambers. ${ }^{13}$

Differentiating between tumor and thrombi. Thrombi were differentiated from tumors using LGE. Thrombus does not have contrast uptake and therefore appears dark on LGE, with surrounding area of high uptake from adjoining blood or myocardium. Thrombus imaging was further enhanced with long-term inversion imaging, which renders thrombus black.

Lipomas and lipomatous hypertrophy. Lipomas and lipomatous hypertrophy have distinct CMR characteristics that are nearly pathognomonic. Lipomas and lipomatous hypertrophy have no soft tissue component and have homogenous increased intensity on T-1-weighted images that decrease completely with fat-saturated sequences. The lipomatous hypertrophy lacks an external capsule, whereas lipomas are well encapsulated.

Tissue composition. The gross morphology was assessed on SSFP cine images of the tumor. Cine imaging allows for accurate anatomic assessment, mobility, and hemodynamic consequences of the tumor. SSFP cine slices were acquired in multiple orientations covering the extent of the tumor. ${ }^{14}$

Signal intensity in magnetic resonance imaging depends on proton density and $\mathrm{T} 1$ and $\mathrm{T} 2$ relaxation times. Different signal intensity can be emphasized on T1- or T2-weighted images to differentiate tumor types. On the other hand, FPP, a standard rest perfusion sequence of gadolinium-based contrast agent, determines vascularity of the tumor. LGE is acquired 10 to 15 minutes after administration of contrast agent. This augments the difference between the tissue and the blood pool. ${ }^{14}$

Tissue characterization of tumors was performed by precontrast ( $\mathrm{T} 1$ and T2 TSE with and without fat suppression), FPP (gradient echo saturation recovery), and postcontrast (delayed hyperenhancement) sequences. T1 and T2 TSE images were analyzed for presence of fat suppression and qualitative signal intensity comparison with left myocardium. The signal intensity was graded as hypointense, isointense, or hyperintense to left ventricular myocardium. FPP was graded as no FPP, hypoperfusion, isoperfusion, and hyperperfusion in comparison with the left ventricular myocardium. Delayed hyperenhancement images were assessed for presence and absence of hyperenhacement. ${ }^{5,15}$

\section{Statistical Methods}

Differences between categoric variables were evaluated with the Fisher exact test. For continuous variables, the Wilcoxon rank-sum test for independent samples was used to determine differences for non-normally distributed continuous variables. The Student $t$ test was used for normally distributed continuous variables. The Shapiro-Wilk test was used to test for normality of continuous variables. The overall goal of the analysis was to describe difference in CMR characteristics of cardiac tumors. However, we 


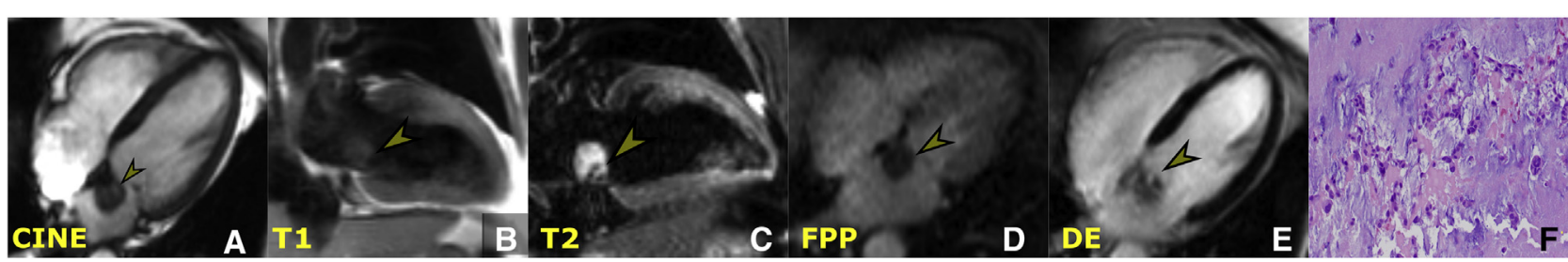

Panel 2: Sarcoma

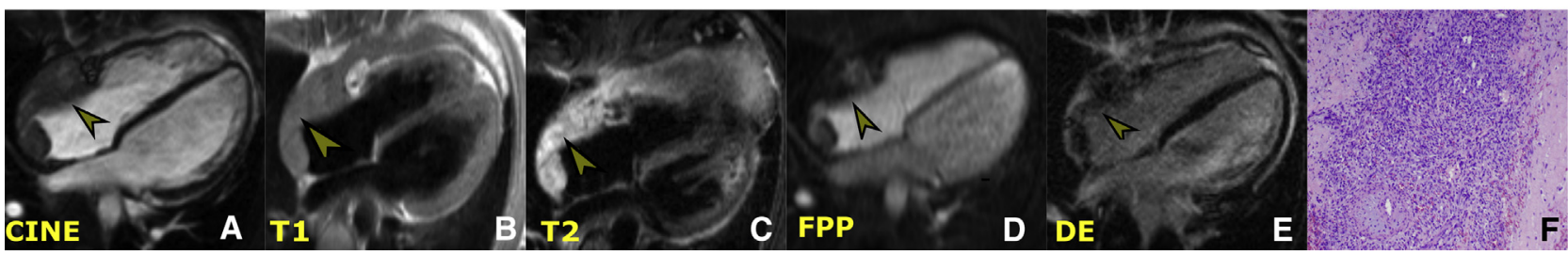

FIGURE 2. Panel 1, Representative images for myxoma attached to the inter-atrial septum (A). The mass appears to be isointense to the myocardium on T1 (B) and isointense to the myocardium on T2 (C). There is no contrast uptake on FPP (D), and there is heterogeneous contrast uptake on delayed enhancement images (E). Pathology slide with hematoxylin-eosin stain $(\times 100)$ with cardiac myxoma composed of anastomosing cords of tumor cells in an extensively myxoid background (F). Panel 2, Representative images for right atrial sarcoma with extension into extracardiac structures (A). The mass appears to be isotense to myocardium on T1 (B) and heterogeneous hyperintense to myocardium on T2 (C). There is significant contrast uptake on FPP (D) and delayed hyperenhancement $(E)$. Pathology slide with hematoxylin-eosin stain $(\times 100)$ with high-grade sarcoma with cellular fascicles of spindle cells exhibiting significant pleomorphism (F). FPP, First-pass perfusion; $D E$, delayed enhancement.

also prespecified a logistic regression model for cardiac tumors dichotomized as benign or malignant. Covariables for the model were selected on the basis of biologic plausibility and included the tumor size, wall infiltration, and presence of FPP. Because the cohort size was small and FPP exhibited separation, Firth logistic regression was used. ${ }^{16}$ The model performance was assessed with receiver operating characteristic curve analysis. In addition, we performed classification and regression tree (CART) analysis to develop a decision-making algorithm ( $\mathrm{R}$ package version 1.21). ${ }^{17}$ The parameters defined to predict malignancy included FPP, delayed enhancement, largest 2-dimensional diameter, pericardial effusion, irregular borders, and infiltration in adjacent structures. All statistical analyses were the responsibility of R.C.S. and performed using R version 1.21. We also used random forest modeling to determine the Gini index for each variable to help understand the most important variables for predicting malignancy. ${ }^{18}$ Interobserver variability was performed for the 2 readers. Interclass correlation coefficient (ICC) was used for continuous variables, and Cohen's kappa was used for categoric measures. ${ }^{19,20}$

\section{RESULTS}

Of the 130 patients enrolled in the study (Figure 1), 28 were referred for a mass suspected on echocardiography, but there was no detectable mass by CMR (pseudotumor). In the cohort of true cardiac tumors, 36 were excluded on the basis of preset criteria (Figure 1, Appendix E1). Sixtysix patients with cardiac tumors were identified and included in this analysis.

\section{Imaging Characteristics of Cardiac Tumors}

Of the 66 tumors, $27(41.0 \%)$ were benign and 39 $(59.0 \%)$ were malignant. The breakup of different tumor types is shown in Figure 1. All tumors had a histopathologic diagnosis except 3 lipomas, 6 lipomatous hypertrophy tumors, and 3 malignant tumors. Tissue diagnosis was made for 48 tumors $(72 \%)$ that were fully resected, and the remaining $6(9 \%)$ diagnoses were based on biopsy specimens from the tumor.

Of the 3 cases with metastasis that had no direct cardiac tumor tissue diagnosis, there was clear supporting evidence of the diagnosis. One was renal cell carcinoma with characteristic imaging. The second case was hepatocellular carcinoma with clear extension in the inferior vena cava and right atrium with tissue diagnosis from the liver. The third patient had a history of colorectal cancer with a large area of metastasis to the lung and heart with tissue diagnosis from the lung. Figure 2 shows some representative tumor types of myxoma and sarcoma as a representation for benign and malignant tumors, respectively.

\section{Comparison of Benign and Malignant Tumors}

The baseline demographics in the 2 groups are detailed in Table 1. Patients (aged 65 years [60.0-71.0] vs 51 years [42.8-60.0], $P<.001$ ) with benign tumors were more likely to have arrhythmias $(P=.04)$, coronary artery disease $(P=.02)$, and higher ejection fraction $(P=.016)$.

The morphologic imaging characteristics were significantly different between malignant and benign tumors (Table 2). Malignant tumors were more likely to have irregular borders $(29[80.0 \%]$ vs $7[26 \%], P<.001)$ and 
TABLE 1. Baseline demographics

\begin{tabular}{lccccc}
\hline \multicolumn{1}{c}{ Factor } & All tumors $=\mathbf{6 6}$ & Malignant $=\mathbf{3 9}$ & Benign $=\mathbf{2 7}$ & $\boldsymbol{P}$ value & Shapiro-Wilk test* \\
\hline Age y (median) & $58.0(45.0-66.0)$ & $51.0(42.8-60.0)$ & $65.0(60.0-71.0)$ & $<.001$ \\
Sex (male) & $36(54.5 \%)$ & $22(56.4 \%)$ & $14(51.9 \%)$ & .779 \\
\hline Smoker & $20(30.3 \%)$ & $10(25.6 \%)$ & $10(37 \%)$ & .154 & .022 \\
Congestive heart failure & $4(6.1 \%)$ & $1(2.6 \%)$ & $3(11.1 \%)$ & .138 & .548 \\
Valvular heart disease & $3(4.5 \%)$ & $1(2.6 \%)$ & $2(7.4 \%)$ & .028 & .254 \\
Coronary heart disease & $10(15.2 \%)$ & $3(7.7 \%)$ & $7(25.9 \%)$ & .041 \\
Hypertension & $23(34.8 \%)$ & $12(30.8 \%)$ & $11(40.7 \%)$ & .347 \\
Arrhythmias & $11(16.7 \%)$ & $4(10.3 \%)$ & $7(25.9 \%)$ & 1 & .016 \\
Diabetes mellitus & $5(7.6 \%)$ & $2(5.1 \%)$ & $3(11.1 \%)$ & $4(14.8 \%)$ & .011 \\
History of malignancy & $10(15.2 \%)$ & $6(15.4 \%)$ & $70.5(60.2-72.8)$ & $18(66.7 \%)$ & 0.035 \\
Ejection fraction (median) & $67.0(59.0-70.8)$ & $64.5(57.8-69.0)$ & $38(97 \%)$ & & \\
Biopsy proven & $56(84.8 \%)$ & & & \\
\hline
\end{tabular}

*Comparison between medians.

to infiltrate into the free wall $(21[53.8 \%]$ vs $0[0 \%]$, $P<.001)$ and adjacent structures $(19[48.7 \%]$ vs $0[0 \%]$ $P<.001)$. Concurrent pericardial effusion was seen more often in patients with malignant tumors (16 [41.0\%] vs 2 [7.4\%], $P=.004)$. Malignant tumors were generally larger than benign tumors (5.0 vs $3.0 \mathrm{~cm}$, respectively, $P=.018$ ), but some myxomas were larger than $5 \mathrm{~cm}$. The distribution of tumor sizes is shown in Figure 3. Although not statistically significant, a greater proportion of malignant tumors were right sided compared with benign tumors (21 [67.7\%] vs $11[42.3 \%], P=.066)$.

In terms of tissue characterization (Table 3), T1- and T2-weighted imaging were not helpful in differentiating benign from malignant tumors. Evidence of visually identifiable FPP was present in all malignant tumors, but only in a few benign tumors (39 [100\%] vs 9 [33.3\%], $P<.001$ ), and seemed to be more homogenously distributed in such tumors $(14[35.9 \%]$ vs $6[22.2 \%], P<.001)$. Likewise, LGE was present in all malignant tumors, but not always in benign tumors $(39[100 \%]$ vs 16 [59.3\%], $P<.001)$.

The sensitivity, specificity, and diagnostic accuracy of tumor CMR features are reported in Table 4. FPP, irregular borders, and infiltration of free wall or adjacent structures had the highest diagnostic accuracy in differentiating benign from malignant tumors $(86.4 \%, 80.3 \%$, and

TABLE 2. Comparison of morphologic features between benign and malignant tumors

\begin{tabular}{|c|c|c|c|c|}
\hline & All tumors $=66$ & Malignant $=\mathbf{3 9}$ & Benign $=27$ & $P$ value \\
\hline \multicolumn{5}{|l|}{ Dimensions } \\
\hline Largest dimension $(\mathrm{cm})$ & $3.8(2.7-6.0)$ & $5.0(3.1-6.3)$ & $3.0(1.8-3.9)$ & .018 \\
\hline Smallest dimension $(\mathrm{cm})$ & $2.4(1.7-4.2)$ & $3.2(1.9-5.0)$ & $2.0(1.5-2.8)$ & .032 \\
\hline \multicolumn{5}{|l|}{ Borders } \\
\hline Well circumscribed & $28(42.4 \%)$ & $7(17.9 \%)$ & $21(77.8 \%)$ & $<.001$ \\
\hline \multicolumn{5}{|l|}{ Location } \\
\hline Aorta & $2(3.0 \%)$ & $1(2.6 \%)$ & $1(3.7 \%)$ & \\
\hline Left atrium & $24(36.4 \%)$ & $10(25.6 \%)$ & $14(51.9 \%)$ & \\
\hline Left ventricle & $4(6.0 \% \%)$ & $3(7.7 \%)$ & $1(3.7 \%)$ & \\
\hline Right atrium & $27(40.9 \%)$ & $18(46.2 \%)$ & $9(33.3 \%)$ & \\
\hline Right ventricle & $5(7.6 \%)$ & $3(7.7 \%)$ & $2(7.4 \%)$ & \\
\hline RVOT & $4(6.0 \%)$ & $4(10.3 \%)$ & $0(0 \%)$ & \\
\hline Left sided & $30(45.4 \%)$ & $14(35.9 \%)$ & $16(59.3)$ & .066 \\
\hline \multicolumn{5}{|l|}{ Infiltration } \\
\hline Free wall & $21(31.8 \%)$ & $21(53.8 \%)$ & $0(0 \%)$ & $<.001$ \\
\hline Invasion in adjacent structures & $19(30.3 \%)$ & $19(48.7 \%)$ & $0(0 \%)$ & $<.001$ \\
\hline \multicolumn{5}{|l|}{ Pericardial effusion } \\
\hline Present & $18(27.3 \%)$ & $16(41 \%)$ & $2(7.4 \%)$ & .004 \\
\hline
\end{tabular}

RVOT, Right ventricular outflow tract. 


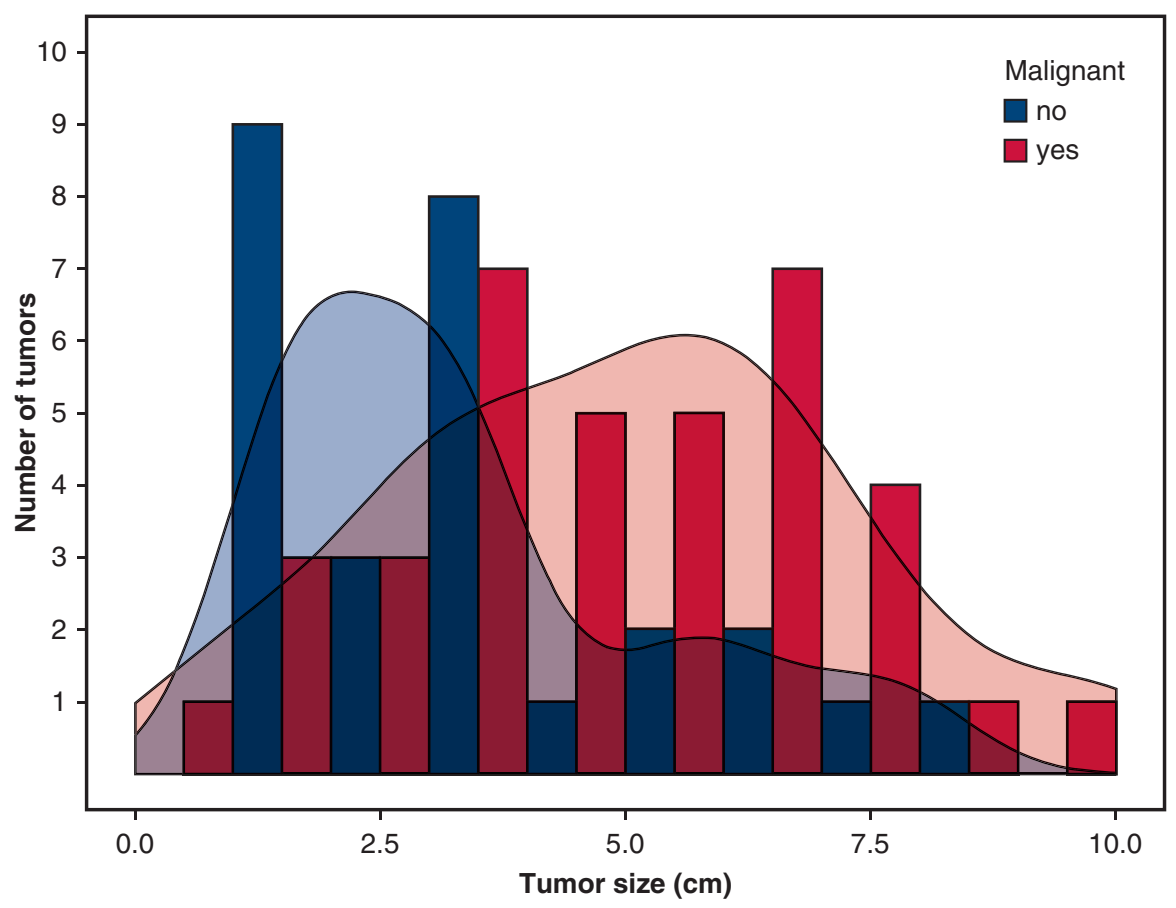

FIGURE 3. Size distribution for benign and malignant tumors.

$89.1 \%$, respectively). Both FPP and LGE had a sensitivity of $100 \%$ because all malignant tumors demonstrated these features. The interobserver variability is shown in Table 5 .

With logistic regression modeling, both FPP $(P<.001)$ and tumor infiltration of the free wall or adjacent structures $(P<.001)$ were independently associated with malignancy
(Table 6). The overall model performed well in discriminating tumors from benign and malignant $(P<.001$, area under the curve $95.5 \%$, Figure E1). However, there are still some limitations, especially in discriminating myxomas (Figure E1) because some cardiac myxomas can be large. In our cohort, the median myxoma

TABLE 3. Comparison of tissue characteristics of benign and malignant tumors

\begin{tabular}{|c|c|c|c|c|}
\hline & All tumors $=66$ & Malignant $=\mathbf{3 9}$ & Benign $=27$ & $P$ value \\
\hline \multicolumn{5}{|l|}{ T1W-TSE } \\
\hline Hyperintense & $16(24.2 \%)$ & $3(7.7 \%)$ & $13(48.1 \%)$ & \\
\hline Hypointense & $3(4.5 \%)$ & $3(7.7 \%)$ & $0(0 \%)$ & \\
\hline Isointense & $47(71.2 \%)$ & $33(84.6 \%)$ & $14(51.9 \%)$ & \\
\hline \multicolumn{5}{|l|}{ T2W-TSE } \\
\hline Hyperintense & $46(69.7 \%)$ & $32(82.1 \%)$ & $14(51.9 \%)$ & \\
\hline Hypointense & $12(18.2 \%)$ & $2(5.1 \%)$ & $10(37 \%)$ & \\
\hline Isointense & $8(12.1 \%)$ & $5(12.8 \%)$ & $3(11.1 \%)$ & \\
\hline \multicolumn{5}{|l|}{ FPP } \\
\hline Present & $48(72.7 \%)$ & $39(100 \%)$ & $9(33.3 \%)$ & $<.001$ \\
\hline Homogenous & $20(30.3 \%)$ & $14(35.9 \%)$ & $6(22.2 \%)$ & $<.001$ \\
\hline \multicolumn{5}{|l|}{ FPP grades } \\
\hline Hyperperfused & $6(9.1 \%)$ & $6(15.4 \%)$ & $0(0 \%)$ & \\
\hline Hypoperfused & $9(13.6 \%)$ & $5(12.8 \%)$ & $4(14.8 \%)$ & \\
\hline Isoperfused & $33(50 \%)$ & $28(71.8 \%)$ & $5(18.5 \%)$ & \\
\hline No perfusion & $18(27.3 \%)$ & $0(0 \%)$ & $18(66.7 \%)$ & \\
\hline \multicolumn{5}{|c|}{ Delayed enhancement } \\
\hline Present & $55(83.3 \%)$ & $39(100 \%)$ & $16(59.3 \%)$ & $<.001$ \\
\hline
\end{tabular}

TIW-TSE, T1-Weighted turbo spin echo; T2-TSE, T2-weighted turbo spin echo; $F P P$, first-pass perfusion. 
TABLE 4. Sensitivity, specificity, and diagnostic accuracy of various cardiac magnetic resonance characteristics

\begin{tabular}{|c|c|c|c|c|c|c|c|}
\hline CMR features & Malignant & Benign & Sensitivity & Specificity & PPV & NPV & $\begin{array}{l}\text { Diagnostic } \\
\text { accuracy }\end{array}$ \\
\hline \multicolumn{8}{|l|}{ Delayed enhancement } \\
\hline+ & 39 & 16 & $\begin{array}{l}100.0 \% \\
\quad(86.8 \%-100.0 \%)\end{array}$ & $\begin{array}{l}40.7 \% \\
\quad(22.4 \%-61.2 \%)\end{array}$ & $\begin{array}{l}70.9 \% \\
\quad(57.1 \%-82.4 \%)\end{array}$ & $\begin{array}{l}100.0 \% \\
\quad(61.5 \%-100.0 \%)\end{array}$ & $\begin{array}{l}75.8 \% \\
\quad(63.6 \%-85.5 \%)\end{array}$ \\
\hline- & 0 & 11 & & & & & \\
\hline \multicolumn{8}{|l|}{ FPP } \\
\hline+ & 39 & 9 & $\begin{array}{l}100.0 \% \\
\quad(86.8 \%-100.0 \%)\end{array}$ & $\begin{array}{l}66.7 \% \\
\quad(46.0 \%-83.5 \%)\end{array}$ & $\begin{array}{l}81.2 \% \\
\quad(67.4 \%-91.1 \%)\end{array}$ & $\begin{array}{l}100.0 \% \\
\quad(74.0 \%-100.0 \%)\end{array}$ & $\begin{array}{l}86.4 \% \\
\quad(75.7 \%-93.6 \%)\end{array}$ \\
\hline- & 0 & 18 & & & & & \\
\hline \multicolumn{8}{|l|}{ FP (homogenous) } \\
\hline+ & 14 & 6 & $\begin{array}{l}35.9 \% \\
\quad(21.2 \%-52.8 \%)\end{array}$ & $\begin{array}{l}33.3 \% \\
\quad(7.5 \%-70.1 \%)\end{array}$ & $\begin{array}{l}70.0 \% \\
\quad(45.7 \%-88.1 \%)\end{array}$ & $\begin{array}{l}10.7 \% \\
\quad(2.3 \%-28.2 \%)\end{array}$ & $\begin{array}{l}35.4 \% \\
\quad(22.2 \%-50.5 \%)\end{array}$ \\
\hline- & 25 & 3 & & & & & \\
\hline \multicolumn{8}{|l|}{ Borders (irregular) } \\
\hline+ & 32 & 6 & $\begin{array}{l}82.1 \% \\
\quad(66.5 \%-92.5 \%)\end{array}$ & $\begin{array}{l}77.8 \% \\
\quad(57.7 \%-91.4 \%)\end{array}$ & $\begin{array}{l}84.2 \% \\
\quad(68.7 \%-94.0 \%)\end{array}$ & $\begin{array}{l}75.0 \% \\
\qquad(55.1 \%-89.3 \%)\end{array}$ & $\begin{array}{l}80.3 \% \\
\quad(68.7 \%-89.1 \%)\end{array}$ \\
\hline- & 7 & 21 & & & & & \\
\hline \multicolumn{8}{|l|}{ Infiltration of free wall } \\
\hline+ & 21 & 0 & $\begin{array}{l}53.8 \% \\
\quad(37.2 \%-69.9 \%)\end{array}$ & $\begin{array}{l}100.0 \% \\
\quad(81.7 \%-100.0 \%)\end{array}$ & $\begin{array}{l}100.0 \% \\
\quad(77.2 \%-100.0 \%)\end{array}$ & $\begin{array}{l}60.0 \% \\
\quad(44.3 \%-74.3 \%)\end{array}$ & $\begin{array}{l}72.7 \% \\
\quad(60.4 \%-83.0 \%)\end{array}$ \\
\hline- & 18 & 27 & & & & & \\
\hline \multicolumn{8}{|l|}{$\begin{array}{l}\text { Infiltration of adjacent } \\
\text { structures }\end{array}$} \\
\hline+ & 19 & 0 & $\begin{array}{l}48.7 \% \\
\quad(33.0 \%-64.4 \%)\end{array}$ & $\begin{array}{l}100.0 \% \\
\quad(81.7 \%-100.0 \%)\end{array}$ & $\begin{array}{l}100.0 \% \\
\quad(77.2 \%-100.0 \%)\end{array}$ & $\begin{array}{l}57.4 \% \\
\quad(43.3 \%-71.6 \%)\end{array}$ & $\begin{array}{l}68.2 \% \\
\quad(55.6 \%-79.1 \%)\end{array}$ \\
\hline- & 20 & 27 & & & & & \\
\hline \multicolumn{8}{|c|}{$\begin{array}{l}\text { Infiltration of free wall } \\
\text { and adjacent structures }\end{array}$} \\
\hline+ & 27 & 0 & $\begin{array}{l}69.2 \% \\
\quad(52.4 \%-83.0 \%)\end{array}$ & $\begin{array}{l}100.0 \% \\
\quad(81.7 \%-100.0 \%)\end{array}$ & $\begin{array}{l}100.0 \% \\
\quad(77.2 \%-100.0 \%)\end{array}$ & $\begin{array}{l}69.2 \% \\
\quad(54.7 \%-83.7 \%)\end{array}$ & $\begin{array}{l}80.3 \% \\
\quad(68.7 \%-89.1 \%)\end{array}$ \\
\hline- & 12 & 27 & & & & & \\
\hline \multicolumn{8}{|l|}{ Pericardial effusion } \\
\hline+ & 16 & 2 & $\begin{array}{l}41.0 \% \\
\quad(25.6 \%-57.9 \%)\end{array}$ & $\begin{array}{l}92.6 \% \\
\quad(75.7 \%-99.1 \%)\end{array}$ & $\begin{array}{l}88.9 \% \\
\quad(65.3 \%-98.6 \%)\end{array}$ & $\begin{array}{l}52.1 \% \\
\quad(37.2 \%-66.7 \%)\end{array}$ & $\begin{array}{l}62.1 \% \\
\quad(49.3 \%-73.8 \%)\end{array}$ \\
\hline- & 23 & 25 & & & & & \\
\hline \multicolumn{8}{|l|}{ Size $>5 \mathrm{~cm}$} \\
\hline+ & 19 & 6 & $\begin{array}{l}48.7 \% \\
\quad(32.4 \%-65.2 \%)\end{array}$ & $\begin{array}{l}77.8 \% \\
\quad(57.7 \%-91.4 \%)\end{array}$ & $\begin{array}{l}76.0 \% \\
\quad(54.9 \%-90.6 \%)\end{array}$ & $\begin{array}{l}51.2 \% \\
\quad(35.1 \%-67.1 \%)\end{array}$ & $\begin{array}{l}60.6 \% \\
\quad(47.8 \%-72.4 \%)\end{array}$ \\
\hline- & 20 & 21 & & & & & \\
\hline \multicolumn{8}{|l|}{ Right-sided tumors } \\
\hline+ & 11 & 21 & $\begin{array}{l}42.3 \% \\
\quad(23.4 \%-63.1 \%)\end{array}$ & $\begin{array}{l}32.3 \% \\
\quad(16.7 \%-51.4 \%)\end{array}$ & $\begin{array}{l}34.4 \% \\
\quad(18.6 \%-53.2 \%)\end{array}$ & $\begin{array}{l}40.0 \% \\
\quad(21.1 \%-61.3 \%)\end{array}$ & $\begin{array}{l}36.8 \% \\
\quad(24.4 \%-50.7 \%)\end{array}$ \\
\hline- & 15 & 10 & & & & & \\
\hline
\end{tabular}

$C M R$, Cardiac magnetic resonance; $P P V$, positive predictive value; $N P V$, negative predictive value; $F P P$, first-pass perfusion; $F P$, first pass.

size was $3.2 \mathrm{~cm}$, with one quarter of the myxomas larger than $5.2 \mathrm{~cm}$. The algorithm for computing probability of malignancy based on these CMR parameters is included as Appendix E2.

Furthermore, using CART analysis, we developed a decision tree algorithm (Figure 4) to help differentiate benign from malignant tumors. This decision tree was approximately $90 \%$ accurate in our data. To estimate real-world performance on de novo data, 10 -fold cross validation was done and demonstrated this tree was approximately $80 \%$ accurate for differentiating benign from malignant tumors. The algorithm-weighted cost of misclassifying a malignant tumor as benign was twice that of classifying a benign tumor as malignant. In our 
TABLE 5. Interobserver variability for morphologic and tissue characteristics

\begin{tabular}{|c|c|}
\hline & Interobserver \\
\hline Visualization with/without contrast & $\mathrm{K}=1$ \\
\hline Size $>5 \mathrm{~cm}$ & $\mathrm{~K}=1$ \\
\hline Border & $\mathrm{K}=0.77$ \\
\hline Infiltration of free wall & $\mathrm{K}=0.77$ \\
\hline Infiltration of adjacent structures & $\mathrm{K}=1$ \\
\hline T1w-TSE & $K=0.64$ \\
\hline T2w-TSE & $\mathrm{K}=0.57$ \\
\hline FPP & $\mathrm{K}=0.55$ \\
\hline FPP (homogenous) & $K=0.68$ \\
\hline FPP grading & $\mathrm{K}=0.72$ \\
\hline Delayed enhancement & $\mathrm{K}=1$ \\
\hline Largest 2-dimensional size & $\mathrm{ICC}=0.76(0.23-0.94)$ \\
\hline
\end{tabular}

Gini index, for predicting the most important variable to predict malignancy, FPP seemed to be the most important determinant (Table E2).

\section{DISCUSSION}

CMR is a strong imaging modality for diagnosing cardiac tumors. Echocardiography is the first-line imaging modality for cardiac tumors and has good sensitivity and specificity. Oftentimes, however, normal anatomic structures may be classified as tumors on echocardiography. ${ }^{21}$ This was demonstrated in our study, because numerous patients who were referred for tumor evaluation did not have any evidence of a cardiac mass on CMR. In this study, however, our primary focus was to describe the CMR characteristics of benign and malignant tumors. Our study is perhaps one of the largest series of benign and malignant tumors with pathological diagnosis $(84 \%)$. One other unique feature in our study was that the majority of the malignant tumors were primary cardiac in origin instead of metastasis as seen in some of the prior studies. ${ }^{9,13}$ This is despite the fact that metastatic tumors are far more common than primary cardiac tumor in adults. ${ }^{22}$ This could be secondary to the fact that the Houston Methodist Hospital is a referral center for primary cardiac tumor surgeries. ${ }^{3,23}$ The most common malignant tumors were sarcomas, and the most common benign tumors were myxomas.

In diagnosing a tumor as benign or malignant by CMR, it is important to correlate what is known regarding the histopathology of a tumor to CMR-dependent tissue parameters. For instance T1- or T2-weighted imaging is useful for characterizing fat, fluid, or blood products. LGE is useful for recognizing areas of necrosis or fibrosis, and vascularity can be assessed by FPP. Infiltration can be assessed using high spatial resolution and multiplanar imaging. Tissue characterization, morphology, and tissue and blood perfusion altogether provide information, which help understand the tumor in question. ${ }^{15}$ For instance, in our study we did not require tissue diagnosis to classify a tumor as a lipoma because this diagnosis is fairly clear based on hyperintensity on T1 imaging with subsequent hypointensity on $\mathrm{T} 2$ imaging with fat saturation.

From our analysis, we noted that important differences exist between benign and malignant tumors that can be identified using CMR. Some of these features are intuitive to what is already known of malignant tumors from pathological studies. ${ }^{24}$ In terms of general characteristics, malignant tumors were larger in size, had irregular borders, and were more invasive. The invasive nature of the tumor carried high specificity for malignant tumors $(100.0 \%)$ but was only modestly sensitive $(69.2 \%)$. This important observation demonstrates that not all malignant tumors are invasive, especially in their earlier stages. Some benign tumors have features that may be misclassified as malignant, particularly based on the size because some benign tumors, especially myxomas, may be fairly large. ${ }^{14,15}$

In our study, contrary to previous studies, a right-sided tumor did not necessarily lead to a diagnosis of malignant tumor. ${ }^{13}$ Less than half of the malignant tumors were located in the right atrium, and some benign tumors had an uncommon location (1 myxoma was located in the left ventricle). Therefore, location can be misleading, for example, a sarcoma with myxomatous tissue can be easily misdiagnosed for a myxoma if present in the left atrium.

In our cohort, T1- and T2-weighted imaging did not discriminate benign from malignant tumors. This finding should not disregard the role T1- and T2-weighted sequences in establishing a diagnosis. Important information regarding tissue characteristics is obtained with these

TABLE 6. Multivariable logistic regression model using Firth bias-adjusted estimates

\begin{tabular}{lcccc}
\hline & Penalized estimate & $\boldsymbol{P}$ value & OR $(\mathbf{9 5} \%$ CI) & Standard error \\
\hline Intercept & -5.096 & $<.001$ & $0.006(<0.001-0.085)$ & 1.756 \\
Infiltration (present) & 3.432 & $<.001$ & $30.927(3.509-3730.617)$ & 1.339 \\
Largest 2-dimension (cm) & 0.2175 & .205 & $1.243(0.892-1.835)$ & 0.176 \\
FPP (present) & 4.545 & $<.001$ & $94.144(8.534-\infty)^{*}$ & 1.667 \\
\hline
\end{tabular}

$O R$, Odds ratio; $C I$, confidence interval; FPP, first-pass perfusion. *Infinite OR is consequence of FPP exhibiting separation (all malignant tumors were positive for FPP). 


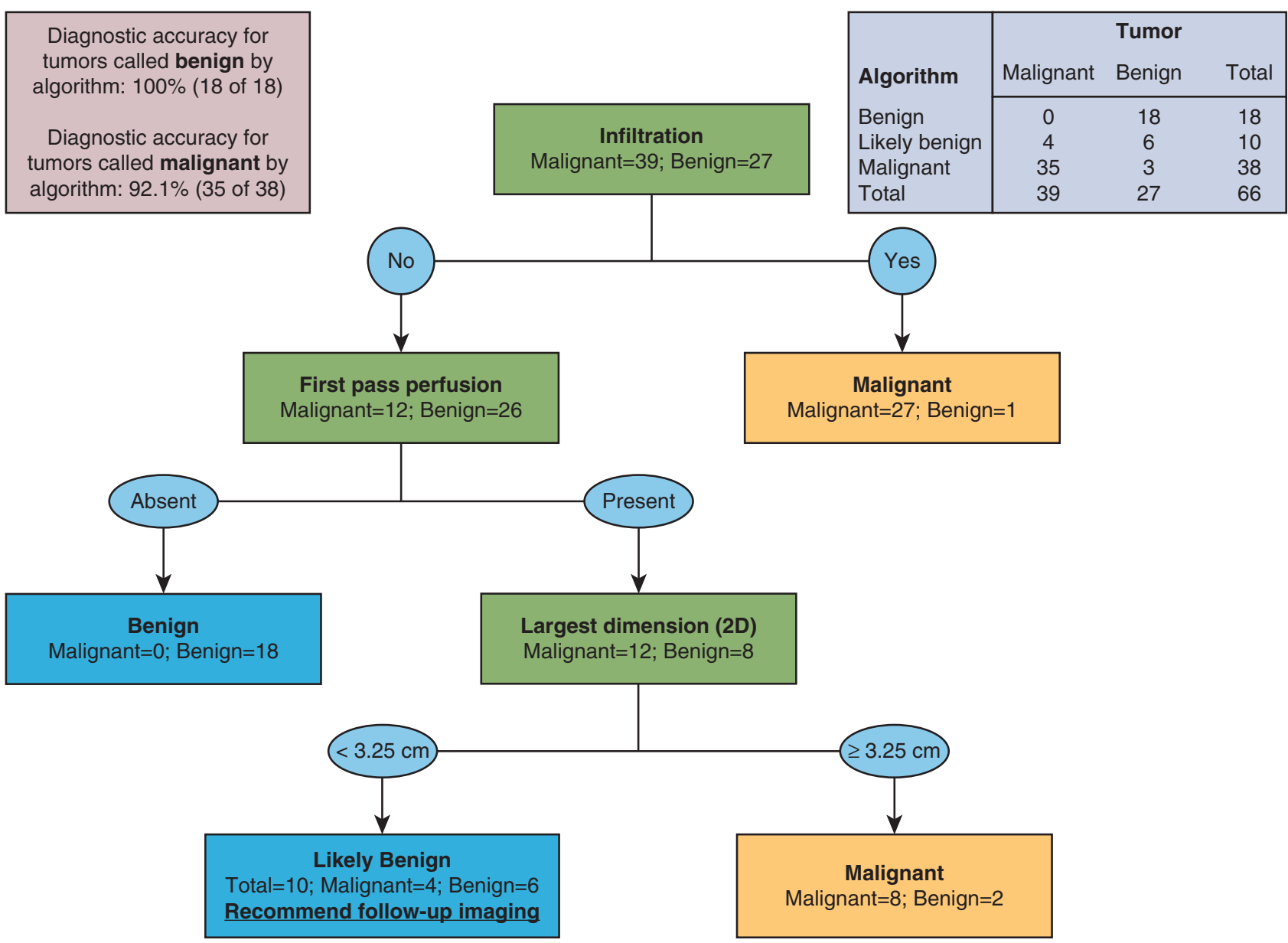

FIGURE 4. Proposed algorithm for tumor diagnosis. 2D, 2-Dimensional.

sequences. Also, in our study and in previously published studies, T1- and T2-weighted imaging is a qualitative measure and perhaps may prove to be more insightful if quantified using newer parametric mapping techniques. ${ }^{25}$

The contrast CMR with FPP and LGE were particularly useful in discriminating benign from malignant tumors. All malignant tumors had presence of FPP as explained by the highly vascular nature of these tumors. LGE was also universally present in all malignant tumors. This could be a result of high rate of cell turnover with higher areas of apoptosis and necrosis in malignant tissues. Some vascular and rapidly growing benign tumors had FPP and LGE; however, the absence of any of these features safely excluded malignancy in our cohort. In our study, FPP carried the highest diagnostic accuracy in diagnosis of malignant tumors $(86.4 \%)$ compared with the study by Pazos-Lopez and colleagues, ${ }^{9}$ in which delayed enhancement had the highest diagnostic accuracy.

Although in our study regression, modeling was limited by sample size and inherent features of the data, we believe there is significant value to the reported analysis. It is clear from the model that both FPP and tumor infiltration are important features of malignant tumors. The strength of our regression modeling approach was that we defined our model using biologic rational before any data analysis (as opposed to training and testing a model on the same small data set, which may result in inherent overfitting). Consequently, the data set used to test model performance was in effect de novo, and this model may perform similarly on future data sets. One caution in interpreting this and any future results is that the cost of misclassifying a benign tumor as malignant is not the same as misclassifying a malignant tumor as benign. In CART analysis, we arbitrarily chose to penalize the cost of misclassifying a malignant tumor as benign twice that of misclassifying a benign tumor as malignant to demonstrate the importance of this in any statistical approach. This imbalance certainly needs to be accounted for in any clinical application of statistical analysis of cardiac tumors. Overall, we suggest our modeling approach be considered an early attempt at 

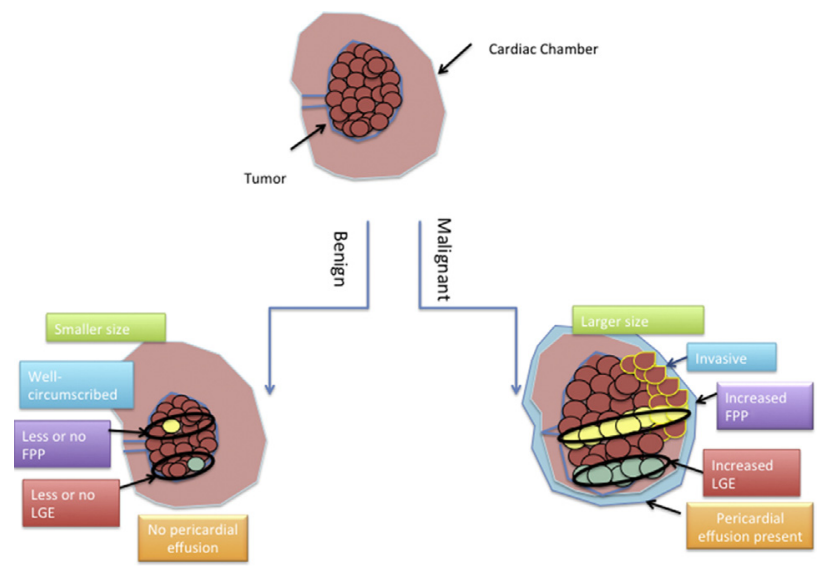

FIGURE 5. Illustration demonstrating differences between benign and malignant tumors based on our proposed algorithm. FPP, First-pass perfusion; $L G E$, late gadolinium enhancement.

an important technique for evaluating cardiac tumors rather than a definitive diagnostic algorithm.

On the basis of our experience with this data set, we would advocate that future modeling approaches include FPP and LGE (both of which are highly sensitive markers for malignant tumors), infiltration, tumor size, and presence of pericardial effusion (all of which tend to be specific for malignant tumors) (Figure 5). Combining multiple imaging parameters into a single estimate using statistical modeling shows significant promise toward noninvasive assessment of cardiac tumors.

\section{Study Limitations}

We recognize that there are several limitations to our study; the first and foremost is the sample size. This particularly affects the negative and positive predictive values in our study because we used the study prevalence for malignant tumors. Although our study involves one of the larger cohorts ever published, we believe that for truly robust analysis, a larger dataset will certainly be required. Given the rarity of cardiac tumors, this begs the need for multicenter collaboration. Second, in our cohort the number of benign tumors was also lower compared with malignant tumors. This is likely due to referral bias to Houston Methodist Hospital. Third, we did not have long-term data on these patients, which could have been insightful in recognizing CMR features that portend poor prognosis, particularly among benign tumors. And last, we think quantifying some of the CMR features including T1- and T2-weighted sequences or FPP may be insightful; however, it may have less applicability for routine clinical practice.

\section{CONCLUSIONS}

Our study demonstrates that CMR is a valuable method for differentiating malignant from benign cardiac tumors. Larger size, irregular borders, invasive nature of the tumor, and presence of pericardial effusion are important morphologic features that help differentiate a malignant tumor from a benign tumor. Contrast imaging with FPP and LGE plays an important role in making this differentiation. However, we recognize that no single feature can be used solely to differentiate benign from malignant tumors. This is due to the inherent tissue difference between different cardiac tumors. Further multicenter studies are needed to confirm our findings.

\section{Conflict of Interest Statement}

Authors have nothing to disclose with regard to commercial support.

\section{References}

1. Abbas A, Garfath-Cox KA, Brown IW, Shambrook JS, Peebles CR, Harden SP. Cardiac MR assessment of cardiac myxomas. Br J Radiol. 2015;88:20140599.

2. Patel SD, Peterson A, Bartczak A, Lee S, Chojnowski S, Gajewski P, et al. Primary cardiac angiosarcoma - a review. Med Sci Monit. 2014;20:103-9.

3. Ramlawi B, Leja MJ, Abu Saleh WK, Al Jabbari O, Benjamin R, Ravi V, et al. Surgical treatment of primary cardiac sarcomas: review of a single-institution experience. Ann Thorac Surg. 2016;101:698-702.

4. Reardon MJ, Malaisrie SC, Walkes JC, Vaporciyan AA, Rice DC, Smythe WR, et al. Cardiac autotransplantation for primary cardiac tumors. Ann Thorac Surg. 2006;82:645-50

5. Hoey ET, Shahid M, Ganeshan A, Baijal S, Simpson H, Watkin RW. MRI assessment of cardiac tumours: part 2, spectrum of appearances of histologically malignant lesions and tumour mimics. Quant Imaging Med Surg. 2014;4:489-97.

6. Esposito A, De Cobelli F, Ironi G, Marra P, Canu T, Mellone R, et al. CMR in assessment of cardiac masses: primary benign tumors. JACC Cardiovasc Imaging. 2014; 7:733-6.

7. Esposito A, De Cobelli F, Ironi G, Marra P, Canu T, Mellone R, et al. CMR in the assessment of cardiac masses: primary malignant tumors. JACC Cardiovasc Imaging. 2014; 7:1057-61.

8. Patel R, Lim RP, Saric M, Nayar A, Babb J, Ettel M, et al. Diagnostic performance of cardiac magnetic resonance imaging and echocardiography in evaluation of cardiac and paracardiac masses. Am J Cardiol. 2016;117:135-40.

9. Pazos-Lopez P, Pozo E, Siqueira ME, Garcia-Lunar I, Cham M, Jacobi A, et al. Value of CMR for the differential diagnosis of cardiac masses. JACC Cardiovasc Imaging. 2014;7:896-905.

10. Weinsaft JW, Kim HW, Shah DJ, Klem I, Crowley AL, Brosnan R, et al Detection of left ventricular thrombus by delayed-enhancement cardiovascular magnetic resonance prevalence and markers in patients with systolic dysfunction. J Am Coll Cardiol. 2008;52:148-57.

11. Fussen S, De Boeck BW, Zellweger MJ, Bremerich J, Goetschalckx K, Zuber M, et al Cardiovascular magnetic resonance imaging for diagnosis and clinical management of suspected cardiac masses and tumours. Eur Heart J. 2011;32:1551-60.

12. Uenishi EK, Caldas MA, Tsutsui JM, Abduch MC, Sbano JC, Kalil Filho R, et al Evaluation of cardiac masses by real-time perfusion imaging echocardiography. Cardiovasc Ultrasound. 2015;13:23.

13. Hoffmann U, Globits S, Schima W, Loewe C, Puig S, Oberhuber G, et al. Usefulness of magnetic resonance imaging of cardiac and paracardiac masses. Am J Cardiol. 2003;92:890-5.

14. Motwani M, Kidambi A, Herzog BA, Uddin A, Greenwood JP, Plein S MR imaging of cardiac tumors and masses: a review of methods and clinical applications. Radiology. 2013;268:26-43.

15. Zhu D, Yin S, Cheng W, Luo Y, Yang D, Lin K, et al. Cardiac MRI-based multi-modality imaging in clinical decision-making: preliminary assessment of a management algorithm for patients with suspected cardiac mass. Int J Cardiol. 2016;203:474-81.

16. Heinze G, Ploner M, Dunkler D, Southworth H. Logistf: Firth's Bias Reduced Logistic Regression. 2013.

17. logistf: Firth's Bias-Reduced Logistic Regression. Available at: http://CRAN.R project.org/package $=$ logistf.

18. Liaw A, Wiener M. Classification and regression by randomForest. $R$ News. $2002 ; 2: 18-22$. 
19. Wolak M, Fairbairn D, Paulsen Y. Guidelines for estimating repeatability. Methods Ecol Evol. 2012;3:129-37.

20. RStudio Team. RStudio: Integrated Development for R. RStudio, Inc., Boston, MA. Available at: http://www.rstudio.com/. 2015.

21. Maybrook RJ, Afzal MR, Parashar S, Deibert B, Chivington M, Walker JY, et al. Intrinsic and extrinsic cardiac pseudotumors: echocardiographic evaluation and review of the literature. Echocardiography. 2016;33:117-32.

22. O'Donnell DH, Abbara S, Chaithiraphan V, Yared K, Killeen RP, Cury RC, et al. Cardiac tumors: optimal cardiac MR sequences and spectrum of imaging appearances. AJR Am J Roentgenol. 2009;193:377-87.
23. Chan EY, Reul RM, Kim MP, Reardon MJ. The "Texas two-step" procedure J Thorac Cardiovasc Surg. 2018;155:285-7.

24. Butany J, Nair V, Naseemuddin A, Nair GM, Catton C, Yau T. Cardiac tumours: diagnosis and management. Lancet Oncol. 2005;6:219-28.

25. Salerno M, Kramer CM. Advances in parametric mapping with CMR imaging. JACC Cardiovasc Imaging. 2013;6:806-22.

Key Words: cardiac magnetic resonance imaging, cardiac tumors, first-pass perfusion 


\section{APPENDIX E1}

\section{Details Regarding Tumors That Were Excluded}

Papillary fibroelastoma $(\mathrm{n}=8)$, unknown diagnosis $(\mathrm{n}=10)$, and thrombi $(\mathrm{n}=18)$ were excluded from the study. Nine of the 10 patients with unknown diagnosis were referral cases from other hospitals, and no clinical or histopathologic data were available. Four patients had malignant features, and 5 patients had benign features based on our ultimately proposed imaging algorithm. One patient underwent biopsy, the results of which were inconclusive.

Of the 10 patients who were excluded, 4 had presumed malignant features and 6 had presumed benign features (based on our ultimately proposed imaging algorithm). Follow-up was performed on these patients with the Social Security Death Index and phone calls. No follow-up was available on 1 presumed malignant and 2 presumed benign tumors. Of the remaining 3 patients with presumed malignant tumors, 2 died and 1 was lost to follow-up after 8 months. None of the patients with benign tumors had died, and the median time to follow-up on benign tumors was 65 months (range, 8-166)

\section{APPENDIX E2}

Presented is an example $\mathrm{R}$ script to calculate the estimated probability of malignancy from the reported logistic regression model. The following example is for a tumor without infiltration, largest tumor diameter of $3 \mathrm{~cm}$, FPP, and hypointense on $\mathrm{T} 1$ and hyperintense on $\mathrm{T} 2$. $\mathrm{R}$ is available as a free statistical analysis software program from http://www.r-project.org/.

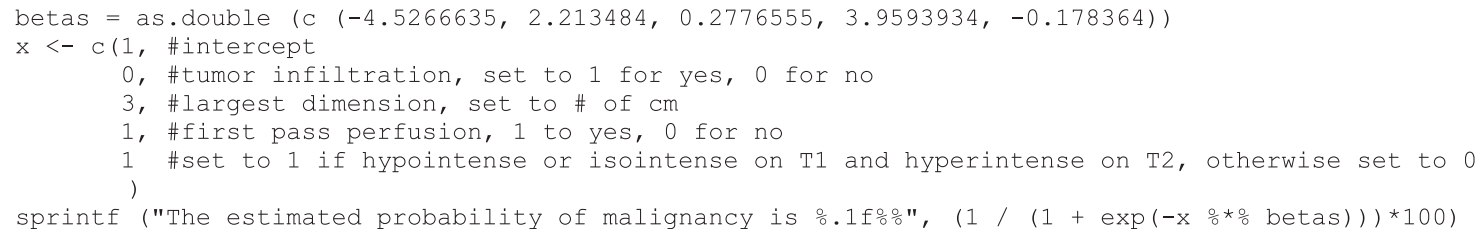




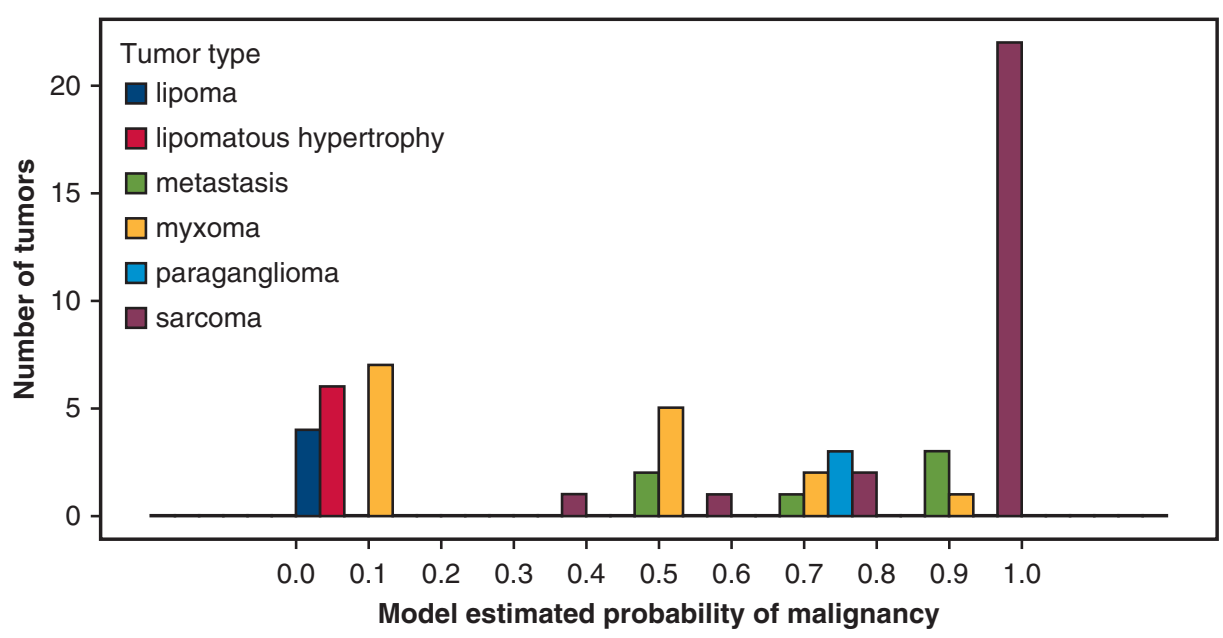

FIGURE E1. Histogram of model estimated probability of malignancy by tumor type.

TABLE E2. Mean decrease in Gini index for selected cardiac magnetic resonance parameters using random forest modeling of 5000 trees with 5 random candidate variables at each split

\begin{tabular}{lc}
\hline & $\begin{array}{c}\text { Mean decrease in Gini } \\
\text { index* }\end{array}$ \\
\hline FPP & 13.0 \\
Largest 2 dimensions & 9.2 \\
Infiltration & 5.0 \\
Borders & 2.5 \\
Pericardial effusion & 1.2 \\
Delayed enhancement & 0.2 \\
\hline
\end{tabular}

$F P P$, First-pass perfusion. *Mean decrease in Gini index obtained using random forest modeling with the package "randomForest" in R (https://cran.r-project.org/ web/packages/randomForest/randomForest.pdf). Algorithm is based on Breiman L. Random forests. Machine Learning. 2001;45:5-32. 